\section{rev Psi}

Revista de Psicología (UNLP)

https://revistas.unlp.edu.ar/revpsi

\title{
¿A qué nos referimos cuando hablamos de tratamiento del autismo? Entrevista a Iván Ruiz
}

\author{
Mariana Calatroni ${ }^{1}$ \\ Correspondencia \\ calatroni.mariana@gmail.com \\ Filiaciones institucionales \\ ${ }^{1}$ Antena Autismo La Plata, Observatorio sobre \\ Políticas del Autismo (FAPOL) (Argentina)
}

\section{Resumen}

La entrevista recorre los atravesamientos actuales en relación a la temática del autismo y se propone profundizar acerca de las particularidades propias de una propuesta de abordaje pensada desde los principios y la ética del psicoanálisis.

\section{Palabras clave \\ psicoanálisis | autismo | tratamiento}

Proceso editorial
$\begin{array}{ll}\text { Recibido } & \text { 1ra decisión } \\ 16 \text { jun. 2020 } & 27 \text { oct. 2020 } \\ \text { Aceptado } & \text { Publicado } \\ \text { 12 jun. 2021 } & 30 \text { jul. 2021 }\end{array}$

Editores

María Cristina Piro (Facultad de Psicología UNLP, Argentina) y Nicolás Alessandroni (Facultad de Psicología UAM, España).
ISSN

2422-572X

Licencia

Licencia de Cultura Libre CC-BY 4.0

(Compartir - Adaptar - Atribuir)

Entidad editora

RevPsi es una publicación de la

Facultad de Psicología (Universidad

Nacional de La Plata, Argentina) 


\section{0 que queremos dizer quando falamos de tratamento do autismo? Entrevista com Iván Ruiz}

\section{Resumo}

A entrevista passa pelas abordagens atuais em relação ao tema do autismo e pretende aprofundar sobre as particularidades de uma proposta de abordagem pensada a partir dos princípios e da ética da psicanálise.

\section{Palavras-chave}

psicoanálise | autismo | tratamento

What do we mean when we talk about autism treatment? Interview with Iván Ruiz

\section{Abstract}

The interview goes through the current approaches in relation to the subject of autism and proposes to deepen about the particularities of a proposal of approach thought from the principles and ethics of psychoanalysis.

\section{Keywords}

psychoanalysis | autism | treatment 


\section{Introducción}

Llueve en Barcelona mientras corren los primeros días de diciembre de 2019. Con cierta prisa y un poco ansiosa llego al consultorio de Iván Ruiz. Iván es psicólogo, tiene su despacho en Barcelona y es director de TEAdir. Ha escrito libros y producido un film documental afines a la temática del Autismo, se ha formado en Psicoanálisis de la Orientación Lacaniana y es Miembro de la Escuela Lacaniana de Psicoanálisis y de la Asociación Mundial de Psicoanálisis.

Nos hemos encontrado en otras oportunidades, pero ésta vez soy yo quién dirige la entrevista. Hemos quedado para que me cuente acerca de TEAdir, la Asociación de padres, madres y familiares de personas con trastorno del espectro autista (Associació de pares, mares i familiars de persones amb trastorn de lespectre autista). La excusa del encuentro es conocer cómo han ido surgiendo los dispositivos singulares que allí ofrecen, qué modalidad de trabajo han ido diseñando y conversar acerca de la actualidad del autismo. Lo que subyace es un trabajo de investigación, en ciernes, que se ha iniciado desde el Laboratorio de Psicoanálisis y Psicopatología de la Facultad de Psicología de la Universidad Nacional de La Plata, y las ganas de empezar a pensar alguna modalidad de abordaje colectivo en el ámbito público que pueda ofrecer una respuesta singular a los requerimientos de niños con padecimiento psíquico en la infancia, sus familias y comunidades afines.

Iván me recibe con serenidad y esa voz apacible. Hablamos en español, si bien en su modo de contar, de hacer silencios y de sostener decididamente algunas ideas, se trasluce el acento catalán, mientras que mis preguntas están plagadas de argentinismos.

\section{Entrevista}

Mariana: Gracias por recibirme Iván. Me gustaría que me cuentes cómo surgió TEAdir, cómo es que se fueron armando los talleres, qué los llevó a pensar así la propuesta, e intentar transmitir algo de eso que, a mi gusto, caracteriza tanto la propuesta que ustedes armaron.

Iván: TEAdir es una asociación de familias, al menos así consta en sus estatutos. Fue en 2010, ahora hace 10 años, que reuní a algunos padres y madres de niños diagnosticados de autismo y, de la mano de algunos colegas psicoanalistas, pusimos en marcha esta asociación. Fue aquél el momento de la celebración del primer Foro sobre autismo, que organizamos desde la Escuela Lacaniana de Psicoanálisis. Estamos en una época en la que las familias se han convertido en un agente político. Las agrupaciones o asociaciones de familias se han convertido en lobbies políticos, entonces no se puede avanzar sin tomar esto en cuenta. Esta situación de entonces -que se mantiene hoy- hizo presente una brecha en lo que eran los circuitos de atención para el autismo. Entendí que teníamos que escuchar que en el autismo se concentraba una nueva presencia del niño y su lazo con el discurso capitalista. Se trataba del niño que debe ser cuidado, al estilo de lo que Lacan dice en su "Nota sobre el niño" cuando se 
refiere al niño que aliena todo acceso a la propia verdad de quien está a su cuidado desde una "exigencia de ser protegido" (Lacan, 1969/2012, p. 394). ¿De qué se quejan las familias? ¿Qué es lo que no funciona en lo han puesto en marcha? Estas preguntas fueron una orientación para mí y lo sigue siendo porque en la queja hay siempre un real que se toca con la punta de los dedos. Es el imposible de toda función educativa como padres pero, en el autismo, es el fracaso que aparece cuando se implementan ayudas para un sujeto que, desde su posición autista, rechaza. El fracaso está entonces en lo real de la posición del autista. Aquel momento inaugural de TEAdir coincidió en Barcelona con un intento de una importante asociación de familias que, con el acuerdo de algunos políticos, pretendió conseguir una legislación para el tratamiento del autismo, proponiendo únicamente métodos reeducativos. $\mathrm{Me}$ pareció, entonces, que debíamos movernos rápido porque eso podía convertirse en un ataque franco contra el psicoanálisis -como así se reveló algunos años después. Así es que movilicé algunas familias que conocía, que confiaban en el trabajo de algunos psicoanalistas, para armar una asociación con representación a nivel social y político, que transmitiera una sensibilidad diferente sobre la cuestión del autismo. Lo que era claro es que hacían falta lugares que no fueran reeducativos, porque toda la vertiente que se dibujaba era una vertiente reeducativa. Se pretendía que fueran las escuelas las que se ocupasen del tratamiento de los niños autistas. Pensé que era el momento de poner en marcha espacios terapéuticos en tiempos de ocio, en los que poder trabajar fuera del marco educativo como tal. La combinación de estos dos significantes funcionó: los tiempos de ocio y el abordaje terapéutico. ¿Por qué los tiempos de ocio? Porque es el tiempo en el que la demanda educativa queda en suspenso. O dicho de otra manera, la demanda hacia el sujeto puede ser amortiguada: Hacia el niño pero también la demanda de los padres hacia sí mismos. Ellos pueden permitirse entonces esperar a lo que su hijo va a hacer cuando no hay la exigencia de responder a los horarios cotidianos, a las obligaciones de la casa, de las tareas escolares, de los cuidados necesarios de sus hijos. Este más allá de lo educativo, ¿no es precisamente la zona en la que se sitúa el psicoanálisis? No es por cualquier razón que Lacan se refirió al discurso del analista como diferente al discurso del amo, en el que encontramos habitualmente situada la educación. Entonces, me pareció que podíamos aprovechar la ocasión para explorar ese margen que se abre cuando damos un giro al discurso educativo y lo llevamos hacia el psicoanálisis.

Mariana: Es bien interesante este margen que planteas. A ver, pensar un dispositivo para los tiempos de ocio en ese margen, ¿sería poder pensar un dispositivo que no necesariamente tenga un sesgo terapéutico?

Iván: (Suspira profundamente) Si no lo llamamos terapéutico, no veo de qué manera podríamos denominarlo. Se trata también de que las familias se sientan concernidas o que los profesionales que derivarán a los chicos intuyan qué tipo de trabajo hacemos. Si nos manejamos con los significantes de la época, la 
atención es o bien educativa o bien es terapéutica. Tampoco me acaba de convencer la denominación de terapéutico. Pusimos en marcha espacios atravesados desde el discurso analítico, lo pensamos de entrada así. A la vez, esto no es incompatible con ofrecer los espacios que sostenemos a partir de significantes que maneja el Otro social. Es de este modo que la oferta que hacemos puede ser escuchada: El Taller de conversación, por ejemplo. ¿Enseñamos a conversar a los niños con autismo que vienen? No, trabajamos con el síntoma del sujeto y sabemos que esa es la consistencia más clara que podemos localizar en ese sujeto. A partir de ahí, evidentemente no vamos a enseñarle a que diga cosas, pero sí estaremos atentos al efecto secundario que se produzca. Si de ahí elementos significantes, incluso palabras... bienvenidas serán.

Mariana: TEAdir ha sido, cómo decirlo, creo... que le encontraron la vuelta, en ese punto. A partir de la experiencia que pude realizar junto a ustedes, una de las cuestiones que me ha llamado la atención es cómo han sabido utilizar las denominaciones de los espacios que ofrecen, tomando por ejemplo el espacio de conversación donde, justamente, el objetivo no es conversar y quienes coordinan ese espacio no tienen ese objetivo como orientación, pero, sin embargo, eso sirve perfectamente de anzuelo, a diferencia de otra orientación donde lo que se ofrece es exactamente lo que se hace.

Iván: Sí. Nuestra oferta es un S2 al S1 que trae el sujeto, por vía propia o por medio de la familia que lo trae. Ahora bien, eso nos compromete porque tenemos, después, que dar cuentas a las familias que traen a sus hijos de qué es lo que hemos hecho alrededor de este significante conversación. Es una cuestión muy delicada: con el trabajo que hacemos con los sujetos, no podemos devolver a la familia un silencio. Eso sería redoblar el silencio que a menudo está en cómo ese sujeto es significado en el discurso de los padres. Digámoslo claro: el autismo de un sujeto hace seriamente difícil instalar un significado en el Otro. Y esto es profundamente político. Es decir que la cuestión política no se acaba, solamente, en la denominación de los espacios sino, después, en cómo explicamos qué es lo que hacemos, cómo lo hacemos transmisible. Diría que esto es un tercer tiempo, nada evidente porque se sostiene en el deseo de cada uno y en cómo TEAdir, como institución, lo favorece.

Mariana: ¿Cómo hacen eso, con los padres, de qué manera?

Iván: Bien, por un lado, los coordinadores de los espacios, de cada dispositivo, mantienen una comunicación con los padres, se encuentran con ellos, se reúnen al principio o al final del tiempo de taller. Pero, además, desde la asociación (TEAdir) hay algunos otros espacios donde mantenemos la posibilidad de que la familia pueda dirigirse a nosotros. Por ejemplo, funcionan dos grupos de padres y madres. Se los llama comúnmente grupos de ayuda mutua. Ahí vienen únicamente los padres -bien, sobre todo las madres. Los invito a hablar y surgen cuestiones muy diversas relativas a sus dificultades en casa, a los impasses en lo cotidiano; pero también hallazgos que han hecho, 
que sirven a otros para preguntarse sobre su posición propia con su hijo. Los padres escuchan de los demás cosas que les permiten pensar que el otro está mucho mejor o mucho peor. Eso favorece un malentendido estructural. El malentendido es que daría la sensación de que vienen a hablar del autismo de sus hijos, pero cada uno habla de su propia condición de sujeto y del punto de real que uno se encuentra con el autismo de su hijo. TEAdir se ha convertido para muchos de ellos en un vínculo nuevo. Se puede ir a TEAdir a hablar de lo que los diagnósticos no llegan a recubrir. De hecho, en el nombre de la asociación, está, en catalán, lo que cada uno té a dir [tiene para decir].

Mariana: O sea, cada uno asiste al grupo voluntariamente, a demanda.

Iván: Eso es.

Mariana: En cuanto a los niños que participan de los dispositivos de TEAdir, ¿tienen, a su vez, un dispositivo individual de tratamiento, con otros profesionales? ¿Cómo se manejan con eso? ¿Cómo articulan?

Iván: La Asociación TEAdir no ofrece en general tratamientos ambulatorios. Los sujetos que vienen tienen sus tratamientos de psicología, de logopedia, educativos, fisioterapia, en otros lugares. Ha sido así desde el inicio aunque eso no obsta que algún momento se lo pueda ofrecer. Pero en ningún caso creo que se trata de entrar en las serie de servicios, públicos o privados, que se dedican al diagnóstico. Eso nos llevaría a participar claramente de la repartición del poder de la atención en el que han entrado muchas entidades, también de padres. Nos situamos por fuera de la lucha por quién se va a quedar con el trozo más grande de pastel. Lo que ofrecemos son espacios de invención en los que trabajamos con varios chicos y varios adultos, -intervinientes, como los llamamos nosotros- en el mismo momento. Y esto, en tiempos de ocio y con un abordaje terapéutico. Haría una salvedad, desde hace unos meses, hemos abierto un espacio por las mañanas para adultos que ya no están en edad de escolaridad obligatoria, a partir de los 16 años. Uno de los proyectos importantes de la Asociación, para muy pronto, va a ser la creación de un Centro Ocupacional, como se llaman aquí, es decir un Centros de Día dónde los chicos a partir de los 16 años y adultos, sobre todo con diagnóstico de autismo pero también con otros diagnósticos de salud mental, puedan venir a ocupar su tiempo. Ya existen Centros así pero éste va a ser un Centro, dentro de la oferta de la Administración de la Generalitat de Catalunya, esa es la apuesta, pero con un trabajo orientado por el psicoanálisis lacaniano.

Mariana: ¡Qué bueno! ¿En función de qué surgió esto?

Iván: Del hecho que hay muchos adultos y adolescentes mayores que se encuentran en situaciones muy complejas, y las familias sin poder ocuparse de ellos más. Los centros a los que en ocasiones asisten parten de una perspectiva reeducativa, aunque hayan acabado la escolaridad. Se mantiene la voluntad de reeducarlos, la misma que los mantenía hasta entonces en una posición de 
infantilización. Buscan su adaptación, su productividad. Entonces algunos de ellos salen rebotados de allí. El espacio que abriremos en TEAdir va a llevar la marca propia del psicoanálisis de Freud, esto es acoger ese real que ha sido expulsado del discurso del amo de las instituciones en las que estaban.

Mariana: Clarísimo. ¿Cómo llegan los niños o jóvenes que ingresarían al centro? ¿Tienen pensado cómo va a ser el modo de entrada? Me refiero a lo que a nivel institucional, en Argentina, solemos llamar "Admisión", que es la instancia a través de la cual se suele conocer a quien realiza una consulta, para ubicar el motivo y darle entrada en un dispositivo; lo que quizás desde el psicoanálisis, más bien, sería trabajar un poco la demanda.

Iván: Sí, desde luego, eso es fundamental si uno se orienta por el psicoanálisis. Ahora tenemos toda la libertad para hacer eso porque no recibimos ayudas públicas. Eso nos da margen para decir "sî" o para decir "no" en función de consideraciones diversas sobre cada caso. De todas maneras, cuando no podemos acoger a un sujeto no le decimos "no", le decimos más bien "qué no". Es decir, que ese "no" lleva un predicado que tiene que ver con la propia demanda de la familia, del sujeto o de la institución que nos lo deriva. Cuando pongamos en funcionamiento este Centro Ocupacional que te mencionaba, tendremos necesariamente que concertarlo económicamente con la Administración para que sea viable. En ese momento tendremos que ver cómo hacemos para que el Discurso del Amo y la burocracia no aplasten, por ejemplo, este periodo necesario para trabajar la demanda. Se trata de localizar aquello que nos dé un signo de que el sujeto podrá incluir la institución en su funcionamiento sinthomático... (Iván hace un silencio y entrecierra los ojos, como si hiciera el esfuerzo de traer una idea que quedó dando vueltas por ahí)... Sobre esto, creo que hay un rasgo propio de TEAdir, del que me he ido dando cuenta con el tiempo.

Mariana: ¿Cuál?

Iván: Con el tiempo lo que se ha ido constituyendo es una especie de anudamiento, diría que un anudamiento sintomático entre, por un lado, la presencia de los padres y las madres, de las familias - porque TEAdir es una Asociación de padres y madres, de familiares, y eso no lo perdemos de vista- y la orientación del psicoanálisis - por el hecho de que mi formación es analítica y también la de los intervinientes, que se forman en la Sección clínica de Barcelona y que se dirigen también a la Escuela Lanicana de Psicoanálisis. Ese anudamiento al que me refiero es lo que me permite mantener un malentendido amable entre la causa de los padres -que es propia de su función como padres- y la causa analítica -que es otra bien distinta. Se trata, sin duda, de una solución encontrada en mi propio análisis y que me permite mi propia función en esta institución. Mi lugar es de Director, no el de psicoanalista, funcionando como una suerte de más uno de un equipo de algo más de 30 personas -entre coordinadores, intervinientes, voluntarios, etc. La función de más uno es 
fundamentalmente la de promover un trabajo de lectura, de reunión, de interrogación de lo que nos gusta denominar la práctica entre varios, en definitiva de las implicaciones que podemos extraer de la enseñanza de Jacques Lacan cuando ésta atraviesa un funcionamiento institucional.

Mariana: ¿Con qué frecuencia son las reuniones?

Iván: Normalmente siempre hay una pequeña reunión cuando termina el taller, habitualmente con una frecuencia semanal. Después hay una reunión de equipo, la reunión de equipo propiamente, que es una vez al mes, y en la que se trabajan algunos casos. Los trabajamos entre varios. Esto hace de la reunión de equipo una reunión muy particular. No es una reunión de equipo como en otras instituciones, es una reunión de equipo orientada por la práctica entre varios, donde se tratan las cuestiones organizativas de cada dispositivo también desde una orientación analítica. Es decir, quién se ocupa de las llaves, del material, quién se encarga de hablar con los padres, de estructurar las reuniones... Pero la reunión de equipo tiene en su centro la pregunta por el sujeto con el que trabajamos. Eso hace de la reunión de intervinientes, no una reunión de expertos sobre el caso, sino de interesados por el enigma del funcionamiento del sujeto. Es un lugar fundamental para nosotros y, debido a la necesidad de disponer de más tiempo para ello, puse en marcha, en el interior del Grupo de Investigación sobre Psicosis y Autismo - en la Sección clínica de Barcelona- un anexo al que llamé Taller de Estudios sobre Práctica Entre Varios. Es un Taller de Estudios vinculado al Grupo de Investigación, aunque son dos espacios diferenciados. Asiste todo aquel que lo desee a la vez que los integrantes de los equipos de los dispositivos de TEAdir. Con lo cual, este nudo sinthomático al que me refería se sostiene también en la formación en psicoanálisis. La experiencia que obtenemos de los dispositivos de TEAdir se revierte en un lugar de formación para poder elaborar qué es esto que llamamos, o lo que se ha convenido en llamar, práctica entre varios, o en todo caso cuál es la práctica entre varios que nosotros hacemos.

Mariana: Exacto. Esa práctica entre varios, que ustedes hacen, es algo que han ido inventando.

Iván: Se podría decir así. Quería llevar a Barcelona lo singular de la práctica que descubrí en Le Courtil, durante el tiempo que estuve allí, pero me vi poniendo en marcha algo con un estilo propio, un estilo que se decanta a partir del deseo de cada uno de los intervinientes que trabajan en TEAdir.

Mariana: Totalmente. Es interesante porque empezamos hablando de la oferta, o de cómo TEAdir fue inventando maneras de poder responder a eso que no entraba en ningún lado ó a lo que nadie podía darle una respuesta... y en el camino se convirtió en un lugar de investigación. Es decir, en el intento de dar respuesta, se desprende una investigación en relación al autismo, en principio, pero también donde se presta la ocasión para que aparezca, y uno se encuentre con, lo sintomático de cada uno. 
Iván: Lo sintomático de cada uno... ¡Sí! ¡Eso no falta! (risas). Se trabaja con el propio sinthome, cuando no con el fantasma. Eso hace de un interviniente diferente a cualquier otro. Lo más complejo de una institución es cómo mantener la pregunta abierta. Porque el fantasma del interviniente tenderá siempre a cerrarla -eso está ya en la propia relación del sujeto con el objeto. Por eso, en TEAdir ponemos a trabajar aquello que se presenta de repente como evidente. ¿Qué entendemos por el tratamiento del autismo? ¿A qué nos referimos con práctica entre varios? ¿Qué es una práctica? ¿De qué se autoriza el interviniente en la práctica entre varios? Si en TEAdir no se promueve una autorización como psicoanalista, porque no hay allí psicoanalistas en tanto tal, ¿de qué se autoriza un interviniente, un coordinador, el Director? Estas preguntas nos llevan de cabeza. Entenderás por qué nos tenemos que referir necesariamente a los textos de Freud, Lacan, entre otros.

Mariana: Esta propuesta, que vos bien detallas, es bien distinta a la propuesta de la reeducación.

Iván: Totalmente distinta. Hay una especie de diccionario en TEAdir. Hay algunos significantes privilegiados con respecto a otros que, eventualmente, ni se usan. No nos escucharás pronunciar la palabra "reeducación", por ejemplo. No usamos "conducta". No porque esté prohibido, sino porque las palabras no son inocentes e indican un lugar de enunciación. Entonces, hay palabras que no se usan y lo transmitimos así a las familias. Cuando es dicho así de claro, las familias aceptan sin problema que trabajemos a partir de eso que el sujeto trae, aquello en lo que está ocupado, en lo que está ya trabajando. Es la idea de Virgino Baio de que el autista está ya al trabajo cuando nos lo encontramos por primera vez. Y, a partir de ahí, la perspectiva que se abre es cómo llevar al sujeto, posicionado en su autismo, al discurso del inconsciente, en el que necesariamente está el vínculo con el Otro. Digamos que es con el discurso del inconsciente que se consigue una metáfora de la posición del sujeto.

Mariana: Los dispositivos, ¿tienen una frecuencia de una vez por semana?

Iván: Sí, habitualmente sí. Aunque las salidas de fin de semana o los campamentos en verano tienen una duración mayor pero no menos frecuencia, claro está.

Mariana: Los que son semanales, ¿Tienen una duración predeterminada o un horario establecido?

Iván: Sí, siempre. El horario es fundamental. El horario y el espacio. Es algo que permite atemperar el capricho del Otro. Por el hecho de que el horario y el espacio están asignados por la dirección de TEAdir $-\mathrm{y}$ no son los intervinientes los que están en disposición de decidirlo- los sujetos que acogemos no interpretan eso como algo dirigido a ellos. Eso no quita que muchos de los cambios que se producen a este nivel vienen dados por peticiones argumentadas que los chicos dirigen al Director.

Mariana: ¿Cómo llegan los sujetos a TEAdir? 
Iván: Normalmente vienen derivados por alguna institución o por algún profesional. O bien, son familias que se han acercado a la Asociación porque están en esta situación difícil con sus hijos y piden ayuda. Eso se presenta de manera dramática en los periodos largos de vacaciones escolares -sobre todo en verano. Es demasiado tiempo para que algunos chicos permanezcan en casa. Han sido expulsados de actividades durante las vacaciones escolares y quedan recluidos en las casas. Es por esta razón que TEAdir empezó su actividad ofreciendo dispositivos para estos chicos en el periodo estival. Organizamos un casal de verano -en el que recibimos a los chicos durante cuatro semanas durante todo el día- y las colonias en el mes de julio -quince días de convivencias con ellos en una casa en la montaña. Esta es, realmente, la experiencia más interesante y más difícil de todas las que hacemos. Ahí está el real de lo que significa pasar un día para un sujeto que vive en lo real. Ahí se juega la dificultad mayor de acompañar a un sujeto autista o psicótico en su cotidiano: los momentos de las comidas, de higiene personal, de ir a dormir. Lo que lo sostiene durante el día, aquello de lo que se protege o que debe evitar a toda costa.

Mariana: Tengo una pregunta más que me planteé a partir de la experiencia en algunos talleres de TEAdir o de los que pude participar en Argentina, en La Cigarra. Tiene que ver con algo que te preguntaba antes respecto de lo terapéutico. Siempre me he preguntado si un espacio de taller podría constituirse como un tratamiento en sí mismo o no, independientemente de los efectos terapéuticos. Y a su vez, si es necesario contar también con un dispositivo de tratamiento individual, o si podría prescindirse de eso como algo necesario. Esto último, tiene que ver con otra pregunta en relación a ¿cómo se leen los efectos de las intervenciones en un dispositivo de más de uno ó desde dónde uno se autoriza para intervenir ahí? ... pero bueno, esa última... (risas).

Iván: Sí, ¡es bien difícil lo que planteas! En realidad, creo que es delicado hablar del tratamiento del autismo. En el momento actual, me parece que no podemos hacer referencia a una cura analítica del autismo. Caeríamos en un error político garrafal, porque el autismo no se cura, ni aquel autismo del llamado autista ni el autismo del goce de un sujeto aislado al final de un análisis. Pero incluso cuando hablamos de tratamiento debemos preguntarnos qué estamos diciendo con eso. Para Melanie Klein se trataba de algo bien particular: un trabajo intensivo de 3 y 4 veces por semana con un sujeto a quien se debía aportar un Edipo del que no disponía. Las cosas, hoy, no van por ahí. La presencia del autismo en la escena política y social hace que el psicoanálisis sea prudente. Con prudente me refiero a que pueda transmitir lo que aporta en la experiencia del uno por uno de los sujetos con los que trabajamos. Pero también la prudencia de que, en el mercado del autismo en el que todo vale, los psicoanalistas deben hacer escuchar lo que el discurso analítico puede aportar a la búsqueda de recursos a la que los padres son empujados. El discurso analítico debe estar allí donde lo real del autismo fracasa. En este sentido, el tratamiento del autismo no es más que 
una invención modesta que anude el síntoma del sujeto autista y el Otro que lo acoge -sea este la familia u otra institución.

Mariana: Tal cual... Bueno, lo dejamos acá. Gracias.

Iván: Gracias a ti.

\section{Conclusión}

Prudencia. Es el significante que me interesa rescatar para empezar a pensar de qué se trata el tratamiento de autismo, desde la perspectiva del psicoanálisis. Prudencia, como un modo de nombrar una perspectiva que no se propone la estandarización de una respuesta terapéutica ni objetivos reeducativos, sino el respeto de la invención singular de cada quién como punto de partida. Pues, la educación y la terapéutica pueden transformarse en algo imposible si no le da lugar a esa singularidad.

Una perspectiva prudente, no obstante, no tiene por qué ser menos potente en su política. Una perspectiva prudente, no ingenua de que la dimensión política siempre está en juego, es necesaria para sostener la invención de un dispositivo que responda a la medida del sujeto, en lugar de elidirlo y segregar aquello que pueda poner el dispositivo en cuestión.

La perspectiva ética que introduce el Psicoanálisis nos permite pensar en la posibilidad de un dispositivo sostenido en principios, no estándares, lo suficientemente flexible, pero no menos riguroso, para que cada quién pueda servirse de él, en lugar quedar reducido a su servidumbre.

\section{Referencias}

Lacan, J. (1969/2012). Nota sobre el niño. En Otros escritos. Paidós. 